WIS-96/6/Jan-PH

hep-th/9601114

\title{
Fusion Rules for Extended Current Algebras
}

\author{
Ernest Baver and Doron Gepner \\ Department of Particle Physics \\ The Weizmann Institute \\ Rehovot 76100 \\ ISRAEL
}

\begin{abstract}
The initial classification of fusion rules have shown that rational conformal field theory is very limited. In this paper we study the fusion rules of extended current algebras. Explicit formulas are given for the S matrix and the fusion rules, based on the full splitting of the fixed point fields. We find that in some cases sensible fusion rules are obtained, while in others this procedure leads to fractional fusion constants.
\end{abstract}




\section{Introduction}

The fusion rule algebra [1, 2, 9] have been studied extensively [2-10], since it seems to play an important role in the classification of the rational conformal field theories. In this paper we concentrate on the fusion rules of the extended chiral algebra, namely: we investigate the fusion rules of the WZW theory [9] on the non-simply connected manifolds $S U(n) / Z_{m}$ at levels*

$$
\begin{array}{ll}
k=n r & \text { for } \mathrm{n} \text { odd, } \\
k=2 n r & \text { for } \mathrm{n} \text { even, }
\end{array}
$$

since for these levels the chiral algebra is an extension of the usual current algebra. Here $Z_{m}$ is the subgroup of the center and $n=m p$, where $\mathrm{p}$ is a positive integer.

The primary fields of the WZW model may be labeled by the highest weight representations of the horizontal Lie algebra. Let the fundamental weights of $S U(n)$ be $\lambda_{i}$. Then the highest weights are given by

$$
\Lambda=\sum_{i=1}^{n-1} m_{i} \lambda_{i},
$$

where $m_{i}$ are nonnegative integers (Dynkin labels). The set of the integrable representations 99 at the level $k$ is denoted by $P_{k}$,

$$
P_{k}=\left\{m_{i} ; 0 \leq \sum_{i} m_{i} \leq k\right\}
$$

Let us denote by $\tilde{S}$, the modular matrix describing the behavior of the characters of the ordinary diagonal $S U(n)_{k}$ theory under modular transformation $\tau \rightarrow-\frac{1}{\tau}$. The fusion coefficients of these models will be denoted by $N_{\Lambda_{1} \Lambda_{2}}^{\Lambda_{3}}$, where $\Lambda_{j} \in P_{k}$. The technic for the calculation of $N_{\Lambda_{1} \Lambda_{2}}^{\Lambda_{3}}$ was developed in [9, 12]. The modular matrix of the corresponding quotient $S U(n) / Z_{m}$ model will be denoted by $S$ without tilde and its fusion coefficients are denoted by $H_{\Lambda_{1} \Lambda_{2}}^{\Lambda_{3}}$. Let $\sigma$ be external automorphism and $C$ charge conjugation whose action on Dynkin labels is defined by:

$$
\begin{aligned}
& \sigma\left(m_{1}, m_{2}, \ldots, m_{n-1}\right)=\left(k-\sum_{j} m_{j}, m_{1}, \ldots, m_{n-2}\right), \\
& C\left(m_{1}, m_{2}, \ldots, m_{n-1}\right)=\left(m_{n-1}, m_{n-2}, \ldots, m_{1}\right)
\end{aligned}
$$

${ }^{*}$ We will denote these levels generically by $k^{*}$ 
The partition function of $S(n)_{k^{\star}} / Z_{m}$ quotient models is given by

$$
Z=\sum_{\substack{\lambda \in P_{k^{\star}} \\ \lambda \text { singlets of } Z_{m}}} \frac{n}{n_{\sigma \lambda}}\left|\sum_{\sigma} \chi_{\sigma \lambda}\right|^{2},
$$

where $\sigma$ is external automorphism, $n_{\sigma \lambda}$ is the length of the orbit generated by the action of $\sigma$ on $\lambda$.

Let $R$ be the fixed point of $\sigma$ i.e. $\sigma R=R$, so that at the levels $k=k^{\star}$ we have:

$$
R=\frac{k^{\star}}{n} \rho,
$$

where $\rho$ is the sum of fundamental weights of $S U(n)$

$$
\rho=\sum_{i=1}^{n-1} \lambda_{i}=\frac{1}{2} \sum_{\alpha>0} \alpha,
$$

where $\alpha$ are the simple roots of $S U(n)$.

For the reasons which will become clear later we will distinguish two cases: i) n-odd, ii) n-even.

\section{Fusion rules of $S U(n)_{n r} / Z_{n}$ for $n$-odd}

As a first example we consider the case when $m=n$ and $n$ is odd prime number.

The generalized characters are given by

$$
c h_{\lambda}=\sum_{\sigma} \chi_{\sigma \lambda}, \quad \lambda \neq R
$$

the corresponding primary field is denoted by $\phi_{\lambda}$, where $\lambda$ is horizontal highest weight of some representative of the orbit generated by the action of $\sigma$ on $\lambda$. The character corresponding to the fixed point $R$ appears in the partition function with the multiplicity $n$, and in order to distinguish between $n$ corresponding primary fields we introduce additional quantum number $j=1, \ldots, n$, these fields are denoted by $\phi_{R_{j}}$.

The modular matrix is given by [10, 11] 


$$
S=\left(\begin{array}{ccccc}
n \tilde{S}_{\mu, \nu} & \tilde{S}_{\mu, R^{1}} & \tilde{S}_{\mu, R^{2}} & \ldots & \tilde{S}_{\mu, R^{n}} \\
\tilde{S}_{R^{1}, \nu} & x & z & \ldots & z \\
\tilde{S}_{R^{2}, \nu} & z & x & \ldots & z \\
\ldots & \ldots & \ldots & \ldots & \ldots \\
\tilde{S}_{R^{n}, \nu} & z & z & \ldots & x
\end{array}\right),
$$

where $\tilde{S}_{\mu, R^{j}}=\tilde{S}_{\mu, R}$. Note that the $S$ matrix given above is restricted to $Z_{n}$-singlets sector i.e. $\mu \lambda_{1}=$ integer.

It is easy to show that $\tilde{S}_{\mu, R}=0$ when $\mu \lambda_{1} \neq$ integer, indeed using the relation 13. $\tilde{S}_{\mu, \sigma \nu}=e^{2 \pi i \mu \lambda_{1}} \tilde{S}_{\mu, \nu}$ one has:

$$
\tilde{S}_{\mu, \sigma R}=e^{2 \pi i \mu \lambda_{1}} \tilde{S}_{\mu, R}=\tilde{S}_{\mu, R}=0, \quad \mu \lambda_{1} \neq \text { integer } .
$$

The previous observation enables us significantly to simplify the calculations: using the corresponding identities for the diagonal $S U(n)_{k^{\star}}$ models we project them onto $Z_{n}$ singlets sector, using Eq.(3). In order to calculate $x$ and $z$ we use the following equations:

$$
(S T)^{3}=S^{2}=C, \quad S S^{\dagger}=1,
$$

where $C$ is charge conjugation matrix $\left(C^{2}=1\right)$ and $T$ is the matrix of the modular transformation $\tau \rightarrow \tau+1$ that is given by

$$
T_{\mu, \nu}=\delta_{\mu, \nu} e^{2 \pi i\left(\Delta_{\mu}-\frac{c}{24}\right)},
$$

where $c$ is the central charge and $\Delta_{\mu}$ is the conformal weight of the primary field $\phi_{\mu}$ which is given by

$$
\Delta_{\mu}=\frac{(\mu, \mu+2 \rho)}{2\left(k^{\star}+n\right)} .
$$

More explicitly from Eqs.(4) we have:

$$
\sum_{\substack{\nu \neq R \\ \nu \lambda_{1}=\text { integer } \\ \nu \bmod \sigma}} n \tilde{S}_{\mu, \nu} \tilde{S}_{\nu, R}=-(x+(n-1) z) \tilde{S}_{\mu, R}=-\tilde{S}_{\mu, R} \tilde{S}_{R, R},
$$

where the second equality is obtained from the similar equation for the diagonal $S U(n)_{k^{\star}}$ theory:

$$
\sum_{\nu \neq R} \tilde{S}_{\mu, \nu} \tilde{S}_{\nu, R}+\tilde{S}_{\mu, R} \tilde{S}_{R, R}=0, \quad \mu \neq R
$$


and the fact that $\tilde{S}_{\mu, R}=0$ when $\mu$ is not a singlet of $Z_{n}$. Now from the Eq.(7) we have

$$
\tilde{S}_{R, R}=x+(n-1) z .
$$

Taking the $R_{j}, R_{l}$ entry in the matrix relation $S^{2}=C$ and assuming that $[C]_{R_{j}, R_{l}}=\delta_{j, l}$ we have:

$$
\begin{aligned}
& \sum_{\substack{\nu \neq R \\
\nu \bmod \sigma}} \tilde{S}_{R, \nu} \tilde{S}_{\nu, R}+x^{2}+(n-1) z^{2}=1, \\
& \sum_{\substack{\nu \neq R \\
\nu \bmod \sigma}} \tilde{S}_{R, \nu} \tilde{S}_{\nu, R}+2 x z+(n-2) z^{2}=0 .
\end{aligned}
$$

From the Eqs.(10-11) it follows that

$$
(x-z)^{2}=1 .
$$

Again taking the $R_{j}, R_{l}$ component in the matrix relation $(S T)^{3}=C$ and subtracting from it the relation which should hold for the diagonal $S U(n)_{k^{\star}}$ theory, namely: $(\tilde{S}, \tilde{T})_{R, R}^{3}=1$ we have:

$$
\tilde{S}_{R, R}^{3}-n \sum_{w, q=1, \ldots, n} S_{R_{j} R_{w}} S_{R_{w} R_{q}} S_{R_{q} R_{l}}=e^{i \pi\left(\frac{c}{4}-6 \Delta_{R}\right)}\left(1-n[C]_{R_{j}, R_{l}}\right)
$$

where $\Delta_{R}$ is the conformal weight of the fields corresponding to the fixed point $\mathrm{R}$ and $\mathrm{c}$ is the central charge:

$$
\begin{gathered}
c=\frac{k\left(n^{2}-1\right)}{(k+n)}=\frac{r\left(n^{2}-1\right)}{(r+1)}, \\
\Delta_{R}=\frac{(R, R+2 \rho)}{2(k+n)}=\frac{r(r+2) \rho^{2}}{2(k+n)}=\frac{r(r+2)\left(n^{2}-1\right)}{24(r+1)},
\end{gathered}
$$

where in the last equality "strange" formula due to Freudenthal-de Vries was used 3 . Using Eqs.(14-15) one may easily show that

$$
e^{i \pi\left(\frac{c}{4}-6 \Delta_{R}\right)}=e^{-i \frac{\pi}{4} r\left(n^{2}-1\right)}=1,
$$

\footnotetext{
${ }^{\dagger}$ Note that the factor $n$ appears in the sum in the Eq.(2.7) when we switch the summation over $\lambda$ to be modulo $\sigma$

${ }^{\ddagger}$ This assumption is confirmed later, see Eq.(13) and below.

${ }^{\S}$ In the case of $S U(n)$ this formula looks like $\rho^{2}=\frac{n\left(n^{2}-1\right)}{12}$
} 
for odd $n$. The left hand side of the Eq.(2.13) may be easily calculated:

$\tilde{S}_{R, R}^{3}-n \sum_{w, q=1, \ldots, n} S_{R_{j} R_{w}} S_{R_{w} R_{q}} S_{R_{q} R_{l}}=\delta_{j, l}(x-z)^{3}+\left(1-\delta_{j, l}\right)(n-1)(z-x)^{3}$,

so that we conclude that $x-z=1$ and $[C]_{R_{j}, R_{l}}=\delta_{j, l}$ as was assumed before. Finally we will write down expressions for $x$ and $z$ :

$$
x=\frac{\tilde{S}_{R, R}+n-1}{n}, \quad z=\frac{\tilde{S}_{R, R}-1}{n} .
$$

The part of fusion rules of the $S U(n)_{n r} / Z_{n}$ quotient models is quite obvious:

$$
\phi_{\nu} \times \phi_{\mu}=\sum_{\substack{\eta \neq R \\ \eta \bmod \sigma}} \sum_{\sigma} N_{\nu \mu}^{\sigma \eta} \phi_{\eta}+N_{\nu \mu}^{R} \sum_{i=1}^{n} \phi_{R^{i}}, \quad \mu, \nu \neq R .
$$

The most interesting part are the fusion rules between the fields corresponding to the fixed point $R$. According to the Verlinde formula [2] the fusion coefficients are given by:

$$
\begin{gathered}
H_{R, R}^{\eta}=\sum_{\substack{\mu \neq R \\
\mu \bmod \sigma}} \frac{\tilde{S}_{R, \mu} \tilde{S}_{R, \mu} \tilde{S}_{\eta, \mu}^{\dagger}}{\tilde{S}_{0, \mu}}+\frac{x^{2}+(n-1) z^{2}}{\tilde{S}_{0, R}} \tilde{S}_{\eta, R}, \\
H_{R, R^{\prime}}^{\eta}=\sum_{\substack{\mu \neq R \\
\mu \bmod \sigma}} \frac{\tilde{S}_{R, \mu} \tilde{S}_{R, \mu} \tilde{S}_{\eta, \mu}^{\dagger}}{\tilde{S}_{0, \mu}}+\frac{2 x z+(n-2) z^{2}}{\tilde{S}_{0, R}} \tilde{S}_{\eta, R},
\end{gathered}
$$

where $\eta \neq R$. From the Eqs. $(12,20,21)$ one may find

$$
\begin{gathered}
H_{R, R}^{\eta}-H_{R, R^{\prime}}^{\eta}=\frac{(x-z)^{2} \tilde{S}_{\eta, R}^{\dagger}}{\tilde{S}_{0, R}}=\frac{\tilde{S}_{\eta, R}^{\dagger}}{\tilde{S}_{0, R}}, \\
H_{R, R}^{\eta}+(n-1) H_{R, R^{\prime}}^{\eta}=\sum_{\substack{\mu \neq R \\
\mu \bmod \sigma}} \frac{n \tilde{S}_{R, \mu} \tilde{S}_{R, \mu} \tilde{S}_{\eta, \mu}^{\dagger}}{\tilde{S}_{0, \mu}}+\frac{(x+(n-1) z)^{2} \tilde{S}_{\eta, R}^{\dagger}}{\tilde{S}_{0, R}}=
\end{gathered}
$$




$$
=\sum_{\substack{\mu \neq R \\ \mu \bmod \sigma}} \frac{n \tilde{S}_{R, \mu} \tilde{S}_{R, \mu} \tilde{S}_{\eta, \mu}^{\dagger}}{\tilde{S}_{0, \mu}}+\frac{\tilde{S}_{R, R}^{2} \tilde{S}_{\eta, R}^{\dagger}}{\tilde{S}_{0, R}}=\sum_{\mu} \frac{n \tilde{S}_{R, \mu} \tilde{S}_{R, \mu} \tilde{S}_{\eta, \mu}^{\dagger}}{\tilde{S}_{0, \mu}}=N_{R, R}^{\eta}, \quad \eta \neq R .
$$

Solving the Eqs. (2.22-2.23) we find

$$
\begin{gathered}
H_{R, R}^{\eta}=\frac{N_{R, R}^{\eta}+f(\eta)(n-1)}{n}, \\
H_{R, R^{\prime}}^{\eta}=\frac{N_{R, R}^{\eta}-f(\eta)}{n},
\end{gathered}
$$

where we defined

$$
f(\eta)=\frac{\tilde{S}_{\eta, R}^{\dagger}}{\tilde{S}_{0, R}}=e^{\frac{2 \pi i}{n}(\eta+2 \rho, \rho)} \prod_{\alpha>0} \frac{1-e^{\frac{2 \pi i}{n}(\eta+\rho, \alpha)}}{1-e^{\frac{2 \pi i}{n}(\rho, \alpha)}}
$$

The rest of the fusion coefficients may be calculated using the same trick:

$$
\begin{gathered}
H_{R, R}^{R}=\sum_{\substack{\mu \neq R \\
\mu \bmod \sigma}} \frac{\tilde{S}_{R, \mu} \tilde{S}_{R, \mu} \tilde{S}_{R, \mu}^{\dagger}}{n \tilde{S}_{0, \mu}}+\frac{x^{3}+(n-1) z^{3}}{\tilde{S}_{0, R}} \\
H_{R, R^{\prime}}^{R^{\prime \prime}}=\sum_{\substack{\mu \neq R \\
\mu \bmod \sigma}} \frac{\tilde{S}_{R, \mu} \tilde{S}_{R, \mu} \tilde{S}_{R, \mu}^{\dagger}}{n \tilde{S}_{0, \mu}}+\frac{3 x z^{2}+(n-3) z^{3}}{\tilde{S}_{0, R}}, \\
H_{R, R}^{R^{\prime}}=\sum_{\substack{\mu \neq R \\
\mu \bmod \sigma}} \frac{\tilde{S}_{R, \mu} \tilde{S}_{R, \mu} \tilde{S}_{R, \mu}^{\dagger}}{n \tilde{S}_{0, \mu}}+\frac{x^{2} z+z^{2} x+(n-3) z^{3}}{\tilde{S}_{0, R}}
\end{gathered}
$$

From Eqs. $(27,28,29)$ we have

$$
\begin{gathered}
3(n-1) H_{R, R}^{R^{\prime}}+(n-2)(n-1) H_{R, R^{\prime}}^{R^{\prime \prime}}+H_{R, R}^{R}= \\
=\sum_{\substack{\mu \neq R \\
\mu \bmod \sigma}} \frac{n \tilde{S}_{R, \mu} \tilde{S}_{R, \mu} \tilde{S}_{R, \mu}^{\dagger}}{\tilde{S}_{0, \mu}}+\frac{\tilde{S}_{R, R}^{3}}{\tilde{S}_{0, R}}=
\end{gathered}
$$

\footnotetext{
"This identity may be proved directly using the expression for the $S$ matrix and the denominator identity $\prod_{\alpha>0}\left(1-e^{-\alpha}\right)^{\text {mult } \alpha}=\sum_{w \in W} \epsilon(w) e^{w(\rho)-\rho}$, where $W$ denotes Weyl group and $\epsilon(w)$ is the sign of Weyl reflection.
} 


$$
\begin{gathered}
=\sum_{\mu} \frac{\tilde{S}_{R, \mu} \tilde{S}_{R, \mu} \tilde{S}_{R, \mu}^{\dagger}}{\tilde{S}_{0, \mu}}=N_{R, R}^{R}, \\
H_{R, R}^{R}-H_{R, R}^{R^{\prime}}=\frac{x+z}{\tilde{S}_{0, R}}, \quad H_{R, R}^{R}-H_{R, R^{\prime}}^{R^{\prime \prime}}=\frac{x+2 z}{\tilde{S}_{0, R}} .
\end{gathered}
$$

Equations (30-31) determine $H_{R_{j}, R_{l}}^{R_{i}}$ completely:

$$
\begin{gathered}
H_{R, R}^{R}=\frac{2-3 n+n^{2}-3 \tilde{S}_{R, R}+3 n \tilde{S}_{R, R}+N_{R, R}^{R} \tilde{S}_{0, R}}{n^{2} \tilde{S}_{0, R}}, \\
H_{R, R}^{R^{\prime}}=\frac{2-n-3 \tilde{S}_{R, R}+n \tilde{S}_{R, R}+N_{R, R}^{R} \tilde{S}_{0, R}}{n^{2} \tilde{S}_{0, R}} \\
H_{R, R^{\prime}}^{R^{\prime \prime}}=\frac{2-3 \tilde{S}_{R, R}+N_{R, R}^{R} \tilde{S}_{0, R}}{n^{2} \tilde{S}_{0, R}} .
\end{gathered}
$$

\subsection{Example: Fusion rules of $S U(3)_{k=3 r} / Z_{3}$}

In this case the condition $\lambda \lambda_{1}=$ integer, translates for the Dynkin labels into:

$$
2 m_{1}+m_{2}=0 \bmod 3 .
$$

The function $f(\eta) \equiv f\left(m_{1}, m_{2}\right)$ defined in Eq.(26) is given by:

$$
f\left(m_{1}, m_{2}\right)=\left\{\begin{array}{ll}
1 & \text { for } m_{1}+m_{2}=3 p \\
0 & \text { for } m_{1}+m_{2}=3 p+1 \\
-1 & \text { for } m_{1}+m_{2}=3 p+2
\end{array},\right.
$$

where $p$ is a non-negative integer. Using the explicit expression for the fusion coefficients of the diagonal SU(3) models [15] one may show that at levels $k=3 r$ :

$$
N_{R, R}^{R}=N_{(r, r) ;(r, r)}^{(r, r)}=r+1 .
$$


By direct calculation one may show

$$
\tilde{S}_{0, R}=\frac{1}{r+1}
$$

Substituting Eqs.(37,38) into Eq.(33) we find:

$$
\begin{gathered}
H_{R, R}^{R^{\prime}}=0, \\
H_{R, R}^{R}=\frac{N_{R, R}^{R}+2 f(R)}{3}, \\
H_{R, R^{\prime}}^{R^{\prime \prime}}=\frac{N_{R, R}^{R}-f(R)}{3} .
\end{gathered}
$$

We checked by computer that this result indeed holds for $k=3,6,9,12,15$

\section{$\mathrm{SU}(\mathbf{3})_{3} / \mathbf{Z}_{3}$}

The partition function is given by:

$$
Z=\left|\chi_{(0,0)}+\chi_{(0,3)}+\chi_{(3,0)}\right|^{2}+3\left|\chi_{(1,1)}\right|^{2},
$$

The correspondence between primary fields and generalized characters is shown below:

$$
\begin{array}{llc}
\phi_{(0,0)} & \leftrightarrow & \chi_{(0,0)}+\chi_{(0,3)}+\chi_{(3,0)} \\
\phi_{(1,1)}, \phi_{(1,1)}^{\prime}, \phi_{(1,1)}^{\prime \prime} & \leftrightarrow & \chi_{(1,1)}
\end{array}
$$

Fusion rules are given by:

$$
\begin{aligned}
\phi_{(1,1)} \times \phi_{(1,1)} & =\mathbf{1} \\
\phi_{(1,1)}^{\prime} \times \phi_{(1,1)}^{\prime} & =\mathbf{1} \\
\phi_{(1,1)}^{\prime \prime} \times \phi_{(1,1)}^{\prime \prime} & =\mathbf{1} \\
\phi_{(1,1)} \times \phi_{(1,1)}^{\prime} & =\phi_{(1,1)}^{\prime \prime}, \text { etc. }
\end{aligned}
$$

$$
\mathrm{SU}(3)_{6} / \mathbf{Z}_{3}
$$

$\mathrm{Z}=\left|\chi_{(0,0)}+\chi_{(0,6)}+\chi_{(6,0)}\right|^{2}+\left|\chi_{(1,1)}+\chi_{(1,4)}+\chi_{(4,1)}\right|^{2}+\left|\chi_{(3,3)}+\chi_{(3,0)}+\chi_{(0,3)}\right|^{2}+3\left|\chi_{(2,2)}\right|^{2}$.

Primary fields: 


$$
\begin{array}{lcc}
\phi_{(0,0)} & \leftrightarrow & \chi_{(0,0)}+\chi_{(0,6)}+\chi_{(6,0)} \\
\phi_{(1,1)} & \leftrightarrow & \chi_{(1,1)}+\chi_{(1,4)}+\chi_{(4,1)} \\
\phi_{(3,3)} & \leftrightarrow & \chi_{(3,3)}+\chi_{(3,0)}+\chi_{(0,3)} \\
\phi_{(2,2)}, \phi_{(2,2)}^{\prime}, \phi_{(2,2)}^{\prime \prime} & \leftrightarrow & \multicolumn{1}{c}{\chi_{(2,2)}}
\end{array}
$$

The fusion rules are given by:

$$
\begin{aligned}
& \phi_{(1,1)} \times \phi_{(1,1)}=1+2 \phi_{(1,1)}+2 \phi_{(3,3)}+\phi_{(2,2)}+\phi_{(2,2)}^{\prime}+\phi_{(2,2)}^{\prime \prime} \\
& \phi_{(1,1)} \times \phi_{(3,3)}=2 \phi_{(1,1)}+\phi_{(3,3)}+\phi_{(2,2)}+\phi_{(2,2)}^{\prime}+\phi_{(2,2)}^{\prime \prime} \\
& \phi_{(1,1)} \times \phi_{(2,2)}=\phi_{(1,1)}+\phi_{(3,3)}+\phi_{(2,2)}^{\prime}+\phi_{(2,2)}^{\prime \prime} \\
& \phi_{(1,1)} \times \phi_{(2,2)}^{\prime}=\phi_{(1,1)}+\phi_{(3,3)}+\phi_{(2,2)}+\phi_{(2,2)}^{\prime \prime} \\
& \phi_{(1,1)} \times \phi_{(2,2)}^{\prime \prime}=\phi_{(1,1)}+\phi_{(3,3)}+\phi_{(2,2)}+\phi_{(2,2)}^{\prime} \\
& \phi_{(3,3)} \times \phi_{(3,3)}=1+\phi_{(1,1)}+\phi_{(3,3)}+\phi_{(2,2)}+\phi_{(2,2)}^{\prime}+\phi_{(2,2)}^{\prime \prime} \\
& \phi_{(3,3)} \times \phi_{(2,2)}=\phi_{(1,1)}+\phi_{(3,3)}+\phi_{(2,2)} \\
& \phi_{(3,3)} \times \phi_{(2,2)}^{\prime}=\phi_{(1,1)}+\phi_{(3,3)}+\phi_{(2,2)}^{\prime} \\
& \phi_{(3,3)} \times \phi_{(2,2)}^{\prime \prime}=\phi_{(1,1)}+\phi_{(3,3)}+\phi_{(2,2)}^{\prime \prime} \\
& \phi_{(2,2)} \times \phi_{(2,2)}=1+\phi_{(3,3)}+\phi_{(2,2)} \\
& \phi_{(2,2)} \times \phi_{(2,2)}^{\prime}=\phi_{(1,1)}+\phi_{(2,2)}^{\prime \prime}, \quad \text { etc. }
\end{aligned}
$$

This fusion ring is equivalent to the fusion ring of the $S U(2)_{16} / Z_{2}$ see Sec.(4.1)

\section{$\mathbf{S U}(\mathbf{3})_{9} / \mathbf{Z}_{3}$}

The partition function is given by

$$
\begin{gathered}
Z=\left|\chi_{(0,0)}+\chi_{(0,9)}+\chi_{(9,0)}\right|^{2}+\left|\chi_{(1,1)}+\chi_{(1,7)}+\chi_{(7,1)}\right|^{2}+\left|\chi_{(2,2)}+\chi_{(2,5)}+\chi_{(5,2)}\right|^{2}+\left|\chi_{(0,3)}+\chi_{(6,0)}+\chi_{(3,6)}\right|^{2}+ \\
\left|\chi_{(3,0)}+\chi_{(0,6)}+\chi_{(6,3)}\right|^{2}+3\left|\chi_{(3,3)}\right|^{2}
\end{gathered}
$$

The primary fields are:

$$
\begin{array}{lcc}
\phi_{(0,0)} & \leftrightarrow & \chi_{(0,0)}+\chi_{(0,9)}+\chi_{(9,0)} \\
\phi_{(1,1)} & \leftrightarrow & \chi_{(1,1)}+\chi_{(1,7)}+\chi_{(7,1)} \\
\phi_{(2,2)} & \leftrightarrow & \chi_{(2,2)}+\chi_{(2,5)}+\chi_{(5,2)} \\
\phi_{(0,3)} & \leftrightarrow & \chi_{(0,3)}+\chi_{(6,0)}+\chi_{(3,6)} \\
\phi_{(3,0)} & \leftrightarrow & \chi_{(3,0)}+\chi_{(0,6)}+\chi_{(6,3)} \\
\phi_{(3,3)}, \phi_{(3,3)}^{\prime}, \phi_{(3,3)}^{\prime \prime} & \leftrightarrow & \multicolumn{1}{c}{\chi_{(3,3)}}
\end{array}
$$


Fusion rules are given by:

$$
\begin{aligned}
& \phi_{(1,1)} \times \phi_{(1,1)}=\mathbf{1}+2 \phi_{(1,1)}+\phi_{(2,2)}+\phi_{(0,3)}+\phi_{(3,0)} \\
& \phi_{(1,1)} \times \phi_{(2,2)}=\phi_{(1,1)}+2 \phi_{(2,2)}+\phi_{(0,3)}+\phi_{(3,0)}+\phi_{(4,4)}+\phi_{(3,3)}+\phi_{(3,3)}^{\prime}+\phi_{(3,3)}^{\prime \prime} \\
& \phi_{(1,1)} \times \phi_{(0,3)}=\phi_{(1,1)}+\phi_{(2,2)}+\phi_{(0,3)}+\phi_{(4,4)} \\
& \phi_{(1,1)} \times \phi_{(3,0)}=\phi_{(1,1)}+\phi_{(2,2)}+\phi_{(3,0)}+\phi_{(4,4)} \\
& \phi_{(1,1)} \times \phi_{(4,4)}=2 \phi_{(2,2)}+\phi_{(0,3)}+\phi_{(3,0)}+2 \phi_{(4,4)}+\phi_{(3,3)}+\phi_{(3,3)}^{\prime}+\phi_{(3,3)}^{\prime \prime} \\
& \phi_{(1,1)} \times \phi_{(3,3)}=\phi_{(2,2)}+\phi_{(4,4)}+\phi_{(3,3)}^{\prime}+\phi_{(3,3)}^{\prime \prime} \\
& \phi_{(2,2)} \times \phi_{(2,2)}=\mathbf{1}+2 \phi_{(1,1)}+5 \phi_{(2,2)}+2 \phi_{(0,3)}+2 \phi_{(3,0)}+5 \phi_{(4,4)}+2\left(\phi_{(3,3)}+\phi_{(3,3)}^{\prime}+\phi_{(3,3)}^{\prime \prime}\right) \\
& \phi_{(2,2)} \times \phi_{(0,3)}=\phi_{(1,1)}+2 \phi_{(2,2)}+\phi_{(0,3)}+\phi_{(3,0)}+2 \phi_{(4,4)}+\phi_{(3,3)}+\phi_{(3,3)}^{\prime}+\phi_{(3,3)}^{\prime \prime} \\
& \phi_{(2,2)} \times \phi_{(3,0)}=\phi_{(1,1)}+2 \phi_{(2,2)}+\phi_{(0,3)}+\phi_{(3,0)}+2 \phi_{(4,4)}+\phi_{(3,3)}+\phi_{(3,3)}^{\prime}+\phi_{(3,3)}^{\prime \prime} \\
& \phi_{(2,2)} \times \phi_{(4,4)}=2 \phi_{(1,1)}+5 \phi_{(2,2)}+2\left(\phi_{(0,3)}+\phi_{(3,0)}\right)+4 \phi_{(4,4)}+2\left(\phi_{(3,3)}+\phi_{(3,3)}^{\prime}+\phi_{(3,3)}^{\prime \prime}\right) \\
& \phi_{(2,2)} \times \phi_{(3,3)}=\phi_{(1,1)}+2 \phi_{(2,2)}+\phi_{(0,3)}+\phi_{(3,0)}+2 \phi_{(4,4)}+\phi_{(3,3)}+\phi_{(3,3)}^{\prime}+\phi_{(3,3)}^{\prime \prime} \\
& \phi_{(0,3)} \times \phi_{(0,3)}=\phi_{(2,2)}+2 \phi_{(3,0)}+\phi_{(4,4)} \\
& \phi_{(0,3)} \times \phi_{(3,0)}=\mathbf{1}+\phi_{(1,1)}+\phi_{(2,2)}+\phi_{(3,3)}+\phi_{(3,3)}^{\prime}+\phi_{(3,3)}^{\prime \prime} \\
& \phi_{(0,3)} \times \phi_{(4,4)}=\phi_{(1,1)}+2 \phi_{(2,2)}+\phi_{(3,0)}+2 \phi_{(4,4)}+\phi_{(3,3)}+\phi_{(3,3)}^{\prime}+\phi_{(3,3)}^{\prime \prime} \\
& \phi_{(0,3)} \times \phi_{(3,3)}=\phi_{(2,2)}+\phi_{(0,3)}+\phi_{(4,4)}+\phi_{(3,3)} \\
& \phi_{(3,0)} \times \phi_{(3,0)}=\phi_{(2,2)}+2 \phi_{(0,3)}+\phi_{(4,4)} \\
& \phi_{(3,0)} \times \phi_{(4,4)}=\phi_{(1,1)}+2 \phi_{(2,2)}+\phi_{(0,3)}+2 \phi_{(4,4)}+\phi_{(3,3)}+\phi_{(3,3)}^{\prime}+\phi_{(3,3)}^{\prime \prime} \\
& \phi_{(3,0)} \times \phi_{(3,3)}=\phi_{(2,2)}+\phi_{(3,0)}+\phi_{(4,4)}+\phi_{(3,3)} \\
& \phi_{(4,4)} \times \phi_{(4,4)}=\mathbf{1}+2 \phi_{(1,1)}+4 \phi_{(2,2)}+2\left(\phi_{(0,3)}+\phi_{(3,0)}\right)+4 \phi_{(4,4)}+\phi_{(3,3)}+\phi_{(3,3)}^{\prime}+\phi_{(3,3)}^{\prime \prime} \\
& \phi_{(4,4)} \times \phi_{(3,3)}=\phi_{(1,1)}+2 \phi_{(2,2)}+\phi_{(0,3)}+\phi_{(3,0)}+2 \phi_{(4,4)}+\phi_{(3,3)}^{\prime}+\phi_{(3,3)}^{\prime \prime} \\
& \phi_{(3,3)} \times \phi_{(3,3)}=\mathbf{1}+\phi_{(2,2)}+\phi_{(0,3)}+\phi_{(3,0)}+2 \phi_{(3,3)} \\
& \phi_{(3,3)} \times \phi_{(3,3)}^{\prime}=\phi_{(1,1)}+\phi_{(2,2)}+\phi_{(4,4)}+\phi_{(3,3)}^{\prime \prime}
\end{aligned}
$$

The fusion rules of $S U(3)_{12} / Z_{3}$ models are given in the appendix.

The graphs whose incidence matrix is equal to $N_{\phi_{(1,1)}}$ are shown at the Fig. [1], where $\phi_{(1,1)}$ corresponds to the character $\chi_{(1,1)}+\chi_{(k-2,1)}+\chi_{(1, k-2)}$. 


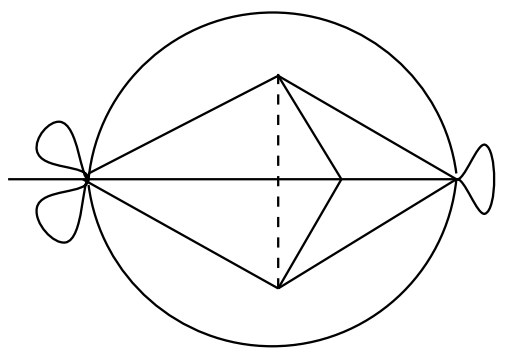

$k=6$

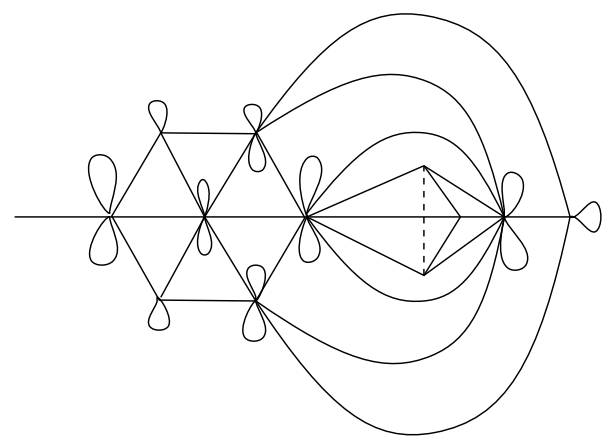

$k=12$

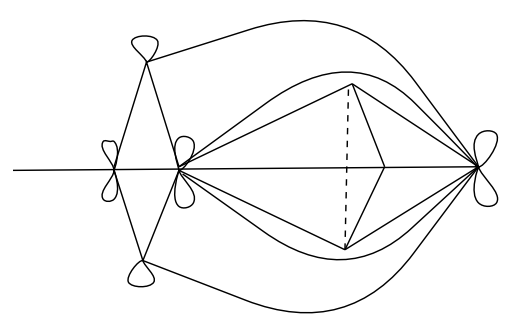

$k=9$

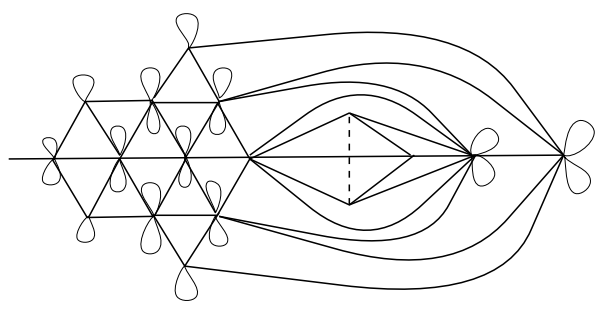

$k=15$

Fig.1: Fusion graphs of the $S U(3)_{k=3 r} / Z_{3}$ models. Here the left free end of the graphs corresponds to the identity operator $\mathbf{1}$, charge conjugation $C\left(m_{1}, m_{2}\right)=\left(m_{2}, m_{1}\right)$ corresponds to the reflection under the horizontal line. The fields $\phi_{R_{j}}, j=1,2,3$ are sitting on the common base of the two pyramids inside the internal circle. 


\section{Generalization for the arbitrary odd $\mathbf{m}$}

If $n$ is not a prime number there is an additional complication which may arise due to appearance of the representations $\zeta$ with

$$
1<n_{\sigma \zeta}<n,
$$

it means that corresponding character will appear in the partition sum with the multiplicity $\frac{n}{n_{\sigma \zeta}}$ and there will be $\frac{n}{n_{\sigma \zeta}}$ primary fields corresponding to the same generalized character $\sum_{\tilde{\sigma} \in Z_{m}} \chi_{\tilde{\sigma} \zeta}$. In this case the matrix $S$ will be given by:

$$
S=\left(\begin{array}{ccc}
m \tilde{S}_{\mu, \nu} & n_{\sigma \zeta} \tilde{S}_{\mu, \zeta_{b}} & \tilde{S}_{\mu, R_{j}} \\
n_{\sigma \zeta} \tilde{S}_{\zeta_{a}, \nu} & y_{a, b} & \frac{n_{\sigma \zeta}}{n} \tilde{S}_{\zeta, R} \\
\tilde{S}_{R_{i}, \nu} & \frac{n_{\sigma \zeta}}{n} \tilde{S}_{R, \zeta} & x_{i, j}
\end{array}\right)
$$

where $a, b=1, . ., \frac{n}{n_{\sigma \zeta}}$ and $i, j=1, \ldots, n$. The fusion rules between primary fields $\phi_{\zeta_{a}}$ may be calculated in a way similar to what was done for the primary fields corresponding to the fixed point $R$. Note that the equations for $x, z$ are the same so that fusion rules between fields $\phi_{R_{i}}$ are remained unchanged.

\section{Fusion rules of $S U(n)_{2 n r} / Z_{m}$ for $n$-even}

For the even $n$ at the level $k=2 n r$ Eq.(2.16) leads us to

$$
e^{i \pi\left(\frac{c}{4}-6 \Delta_{R}\right)}=e^{-i \frac{\pi}{2} r} .
$$

For $r$ even which is divisible by 4 the right hand side of the Eq.(4.1) is equal to 1 , and the result is identical to the discussed in the case of odd $n$. For $r$ even which is not divisible by 4 from Eq.(2.17) one may find $x-z=-1$, and the only difference from the previously discussed result is in the expressions for the $x$ and $z$ which lead to the different expression for the fusion coefficients $H_{R_{j}, R_{l}}^{R_{w}}$,

$$
\begin{aligned}
H_{R, R}^{R} & =\frac{-2+3 m-m^{2}-3 \tilde{S}_{R, R}+3 m \tilde{S}_{R, R}+N_{R, R}^{R} \tilde{S}_{0, R}}{m^{2} \tilde{S}_{0, R}} \\
H_{R, R}^{R^{\prime}} & =\frac{-2+m-3 \tilde{S}_{R, R}+m \tilde{S}_{R, R}+N_{R, R}^{R} \tilde{S}_{0, R}}{m^{2} \tilde{S}_{0, R}} \\
H_{R, R^{\prime}}^{R^{\prime \prime}} & =\frac{-2-3 \tilde{S}_{R, R}+N_{R, R}^{R} \tilde{S}_{0, R}}{m^{2} \tilde{S}_{0, R}}
\end{aligned} .
$$


If $r$ is odd one may show that Eqs.(2.4) do not have solutions for $m>2$. Indeed denoting:

$$
S_{R_{j}, R_{l}}=x_{j, l},
$$

where we anticipate that the naive parameterization of the modular matrix $S$ which we used in Eq.(2.2) (only $x$ and $z$ ) will not work. Using $S^{2}=C$ we have:

$$
\sum_{\zeta=1}^{m} x_{j, \zeta} x_{\zeta, l}=\frac{\tilde{S}_{R, R}^{2}-1}{m}+C_{R^{j}, R^{l}},
$$

plugging this into Eq.(2.4) we obtain:

$$
\tilde{S}_{R, R}-m \sum_{\eta} C_{R^{j}, R^{\eta}} x_{\eta, l}=i(-1)^{\frac{r+1}{2}}\left(1-m C_{R^{j}, R^{l}}\right) .
$$

Calculating $x_{\eta, l}$ from this equation we find:

$$
x_{\eta, l}=\frac{\tilde{S}_{R, R}-i(-1)^{\frac{r+1}{2}}\left(1-m \delta_{\eta, l}\right)}{m} .
$$

Using Eq.(4) and Eq.(6) we get the following equality:

$$
C_{R^{\zeta}, R^{\eta}}=\frac{2}{m}-\delta_{\zeta, \eta}
$$

which shows that the only case which may be treated as reasonable solution is when $m=2$, since when $m \neq 2$ the fusion coefficients $N_{i, j}^{0}$ are fractional.

In order to illustrate what happens we will consider the simplest example corresponding to $S U(2)_{4 r} / Z_{2}$, the generalization for arbitrary even $n$ and $m=2$ is obvious from the previous discussion.

\subsection{Example: $S O(3)_{4 r}=S U(2)_{4 r} / Z_{2}$}

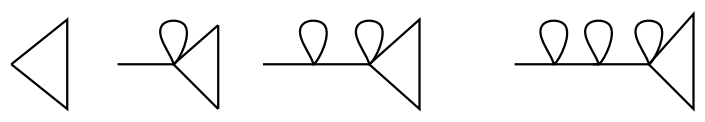

Fig.2: Fusion graphs for $S U(2)_{4 r} / Z_{2}$, whose incidence matrix is given by $N_{\phi_{1}}$ 
In this case the matrix $S$ is given by:

$$
S=\left(\begin{array}{ccc}
2 \tilde{S}_{\mu, \nu} & \tilde{S}_{\mu, R^{1}} & \tilde{S}_{\mu, R^{2}} \\
\tilde{S}_{R^{1}, \nu} & x & z \\
\tilde{S}_{R^{2}, \nu} & z & x
\end{array}\right),
$$

where $x$ and $z$ are given by Eq.(6). Labeling primary fields by their isospin $j=\frac{m_{1}}{2}$ and repeating the same steps as in the Sec.(2) we arrive to the following expression for the function $f\left(m_{1}\right)$ :

$$
f(j)=e^{i \pi j}
$$

The fusion rules are given by:

$$
\begin{aligned}
& \phi_{i} \times \phi_{j}=\sum_{p \neq r}\left(N_{i, j}^{p}+N_{i, j}^{2 r-p}\right) \phi_{p}+N_{i, j}^{r}\left(\phi_{r}+\phi_{r}^{\prime}\right) \\
& \phi_{r} \times \phi_{r}=\sum_{p=0}^{\frac{r}{2}} \phi_{2 p} \quad \mathrm{r}-\text { even } \\
& \phi_{r} \times \phi_{r}^{\prime}=\sum_{p=0}^{\frac{r-2}{2}} \phi_{2 p+1} \quad \mathrm{r}-\text { even } \\
& \phi_{r} \times \phi_{r}=\sum_{p=0}^{\frac{r-3}{2}} \phi_{2 p+1}+\phi_{r}^{\prime} \quad \mathrm{r}-\text { odd } \\
& \phi_{r} \times \phi_{r}^{\prime}=\sum_{p=0}^{\frac{r-1}{2}} \phi_{2 p} \quad \mathrm{r}-\text { odd }
\end{aligned}
$$

For $r=4$ this result was obtained in [14] and it is in agreement with our result up to a type error. Note that the fusion ring when $r=4$ is identical to the fusion ring of $S U(3)_{6} / Z_{3}$.

Note added: While writing this work we received [16] which overlaps part of the discussion here.

\section{Acknowledgments}

We thank S. Cherkis and A. Kapustin for useful discussions.

\section{References}

[1] A.A. Belavin, A.M. Polyakov and A.B. Zamolodchikov, Nucl.Phys. B241(1984)333;

[2] E. Verlinde, Nucl. Phys. B300(1988)360; 
[3] J. Fuchs, Fortschr. Phys. 42(1994)1, hep-th/93060162;

[4] D. Gepner and A. Kapustin, Phys. Let. B349(1995)71;

[5] M. Caselle and G. Ponzano, Phys. Let. B242(1990)52;

[6] M. Caselle and G. Ponzano, Int. J. Mod. Phys. A5(1990)4967;

[7] M. Caselle, G. Ponzano and F. Ravanini, Int. J. Mod. Phys. B6(1992)2075;

[8] W. Eholzer, BONN-TH-94-18, MPI-94-91, Commun. Math. Phys. (to appear)

[9] D. Gepner and E. Witten, Nucl. Phys. B278(1986)493;

[10] V.B. Petkova and J.B. Zuber ASI-TPA-014-95, hep-th/9510175;

[11] A.N. Schellekens and S. Yankielowicz, Nucl. Phys. B334(1990)67; Int. J. Mod. Phys. A5(1990)2903;

[12] D. Gepner, Com. Math. Phys. 141(1991)381;

[13] D. Gepner, Phys. Let. B222(1989)207;

[14] R. Dijkgraaf and E. Verlinde (Utrecht U.), Presented at Annecy Conf. on Conformal Field Theory, Annecy, France, Mar 14-16, 1988;

[15] L. Begin, P. Mathieu and M.A. Walton, Mod. Phys. Lett. A7 (1992)3255;

[16] J. Fuchs, B. Schellekens and C. Schweigert, hep-th/9601078. 


\section{APPENDIX}

\section{$\mathrm{SU}(3)_{12} / \mathrm{Z}_{3}$}

The partition function is given by:

$$
\begin{gathered}
Z=\left|\chi_{(0,0)}+\chi_{(0,12)}+\chi_{(12,0)}\right|^{2}+\left|\chi_{(1,1)}+\chi_{(1,10)}+\chi_{(10,1)}\right|^{2}+\left|\chi_{(2,2)}+\chi_{(2,8)}+\chi_{(8,2)}\right|^{2}+ \\
\left|\chi_{(3,3)}+\chi_{(3,6)}+\chi_{(6,3)}\right|^{2}+\left|\chi_{(0,3)}+\chi_{(3,9)}+\chi_{(9,0)}\right|^{2}+\left|\chi_{(3,0)}+\chi_{(9,3)}+\chi_{(0,9)}\right|^{2}+ \\
\left|\chi_{(1,4)}+\chi_{(4,7)}+\chi_{(7,1)}\right|^{2}+\left|\chi_{(4,1)}+\chi_{(7,4)}+\chi_{(1,7)}\right|^{2}+\left|\chi_{(5,5)}+\chi_{(2,5)}+\chi_{(5,2)}\right|^{2}+ \\
\left|\chi_{(6,6)}+\chi_{(0,6)}+\chi_{(6,0)}\right|^{2}+3\left|\chi_{(4,4)}\right|^{2} .
\end{gathered}
$$

The primary fields are:

$\begin{array}{llc}\mathbf{1} & \leftrightarrow & \chi_{(0,0)}+\chi_{(0,12)}+\chi_{(12,0)} \\ \phi_{1} & \leftrightarrow & \chi_{(1,1)}+\chi_{(1,10)}+\chi_{(10,1)} \\ \phi_{2} & \leftrightarrow & \chi_{(2,2)}+\chi_{(8,2)}+\chi_{(2,8)} \\ \phi_{3} & \leftrightarrow & \chi_{(3,3)}+\chi_{(3,6)}+\chi_{(6,3)} \\ \phi_{4} & \leftrightarrow & \chi_{(0,3)}+\chi_{(3,9)}+\chi_{(9,0)} \\ \phi_{5} & \leftrightarrow & \chi_{(3,0)}+\chi_{(9,3)}+\chi_{(0,9)} \\ \phi_{6} & \leftrightarrow & \chi_{(1,4)}+\chi_{(4,7)}+\chi_{(7,1)} \\ \phi_{7} & \leftrightarrow & \chi_{(4,1)}+\chi_{(7,4)}+\chi_{(1,7)} \\ \phi_{8} & \leftrightarrow & \chi_{(5,5)}+\chi_{(2,5)}+\chi_{(5,2)} \\ \phi_{9} & \leftrightarrow & \chi_{(6,6)}+\chi_{(0,6)}+\chi_{(6,0)} \\ \phi_{10}, \phi_{10}^{\prime}, \phi_{10}^{\prime \prime} & \leftrightarrow & \chi_{(4,4)}\end{array}$




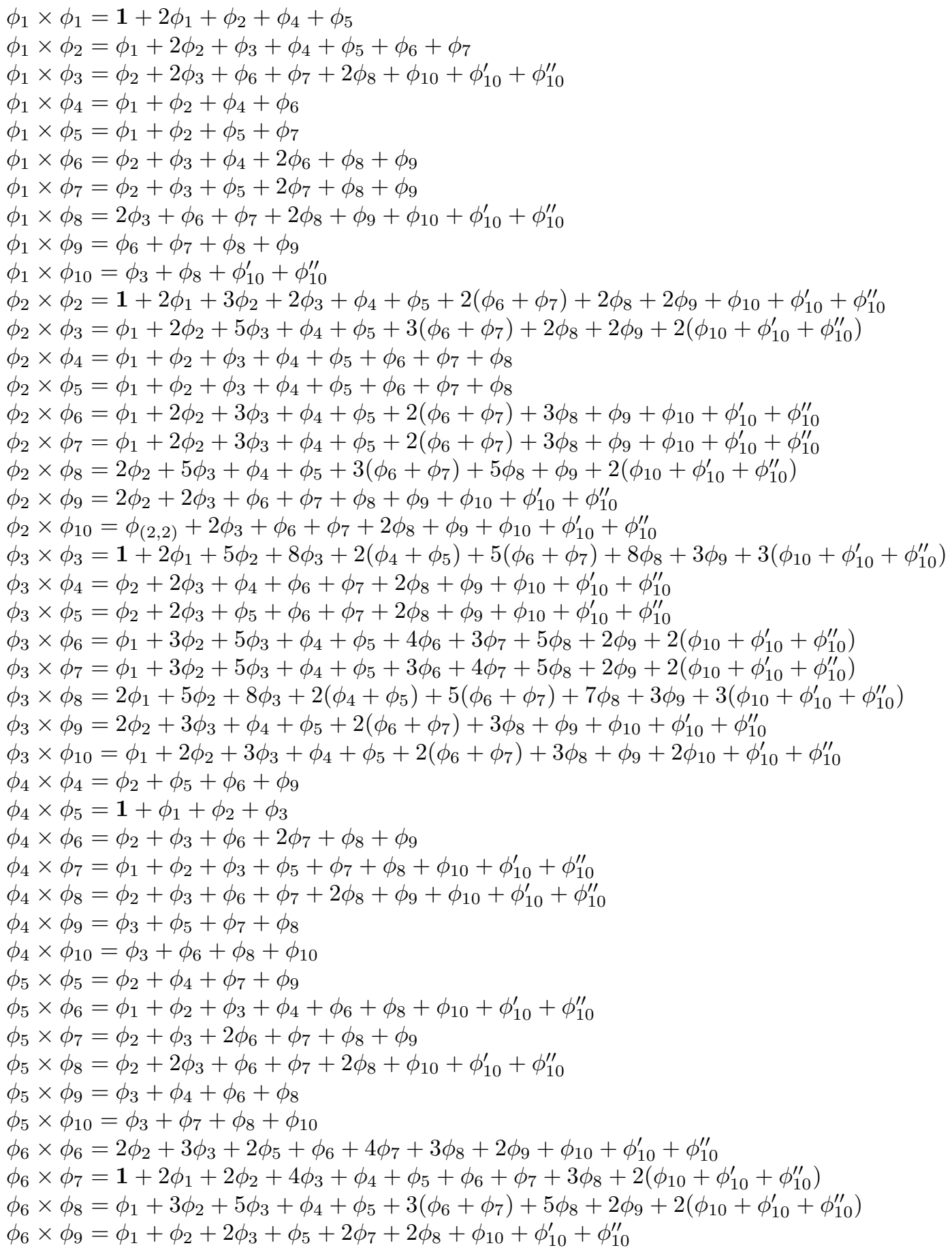




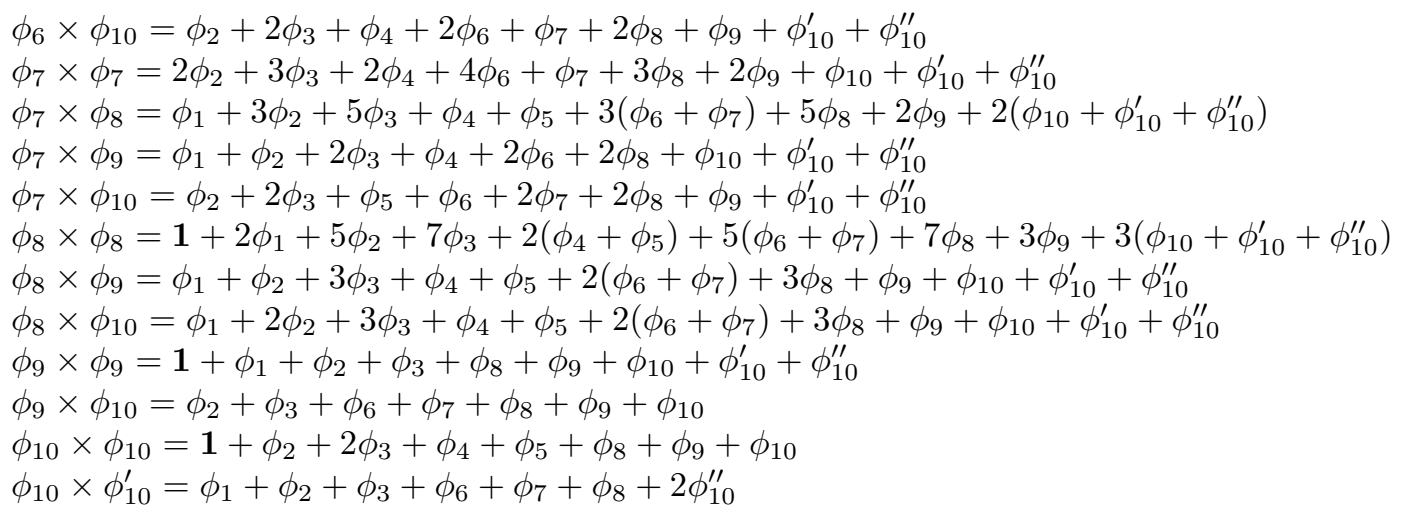

